\title{
Mechanism of corepressor binding and release from nuclear hormone receptors
}

\author{
Laszlo Nagy, ${ }^{3,5,6}$ Hung-Ying Kao, ${ }^{3,6}$ James D. Love, ${ }^{1,4}$ Chuan Li, ${ }^{3}$ Ester Banayo, ${ }^{2,3}$ John T. Gooch, ${ }^{1}$ \\ V. Krishna, K. Chatterjee, ${ }^{4}$ Ronald M. Evans, ${ }^{2,3,7}$ and John W.R. Schwabe ${ }^{1,3,7}$ \\ ${ }^{1}$ Medical Research Council (MRC), Laboratory of Molecular Biology, Cambridge, CB2 2QH, UK; ${ }^{2}$ Howard Hughes Medical \\ Institute (HHMI), ${ }^{3}$ The Salk Institute for Biological Studies, Gene Expression Laboratory, La Jolla, California 92037 USA; \\ ${ }^{4}$ Department of Medicine, University of Cambridge, Addenbrooke's Hospital, Cambridge, CB2 2QQ, UK
}

The association of transcription corepressors SMRT and N-CoR with retinoid and thyroid receptors results in suppression of basal transcriptional activity. A key event in nuclear receptor signaling is the hormone-dependent release of corepressor and the recruitment of coactivator. Biochemical and structural studies have identified a universal motif in coactivator proteins that mediates association with receptor LBDs. We report here the identity of complementary acting signature motifs in SMRT and N-CoR that are sufficient for receptor binding and ligand-induced release. Interestingly, the motif contains a hydrophobic core (ФххФФ) similar to that found in NR coactivators. Surprisingly, mutations in the amino acids that directly participate in coactivator binding disrupt the corepressor association. These results indicate a direct mechanistic link between activation and repression via competition for a common or at least partially overlapping binding site.

[Key Words: Transcription corepressors; SMRT; coactivator binding; corepressor binding; nuclear hormone receptors]

Received September 27, 1999; revised version accepted November 4, 1999.

Members of the steroid hormone receptor superfamily are hormone-activated transcription factors that control vertebrate development, differentiation, and homeostasis through regulating complex gene networks (Mangelsdorf and Evans 1995; Mangelsdorf et al. 1995). Receptors for thyroid hormone and retinoid acid function as potent repressors in the absence of ligand and as activators upon ligand binding. Intensive studies on the mechanisms underlying this regulation led to the identification of different families of proteins that bind to the receptors in the absence and presence of hormone. SMRT (for silencing mediator for retinoid and thyroid hormone receptors) and N-CoR (for nuclear receptor corepressor) are homologous proteins that mediate the repressive effect of unliganded nuclear receptors through the recruitment of histone deacetylase complexes (Alland et al. 1997; Hassig et al. 1997; Heinzel et al. 1997; Laherty et al. 1997; Nagy et al. 1997; Zhang et al. 1997). In contrast, $\mathrm{CBP} / \mathrm{p} 300$, p300/CBP-associated factor (PCAF), and members of the p160 family (SRC-1; GRIP1/ TIF2; activator for thyroid hormones and retinoid recep-

\footnotetext{
${ }^{5}$ Present address: Department of Biochemistry and Molecular Biology, University Medical School of Debrecen, Debrecen, Hungary 4H-012. ${ }^{6}$ These authors contributed equally.

${ }^{7}$ Corresponding authors.

E-MAIL evans@salk.edu; FAX (858) 455-1349.

E-MAIL jws2@mrc-lmb.cam.ac.uk; FAX 441223213556.
}

tor (ACTR)/RAC3/P/CIP) (Onate et al. 1995; Hong et al. 1996; Kamei et al. 1996; Yao et al. 1996; Chen et al. 1997; Torchia et al. 1997; Blanco et al. 1998) possess intrinsic histone acetyl transferase activity and potentiate the transcriptional activity of ligand bound receptors.

Nuclear receptors contain two evolutionarily conserved modules, the DNA binding domain (DBD) and the ligand binding domain (LBD). LBDs are required for nuclear localization, homo- and/or heterodimerization, and most importantly ligand binding and ligand-induced switch of the transcriptional activity. Molecular studies established that the LXXLL signature motif within coactivators confers stereospecific interaction with ligandactivated nuclear receptors (Heery et al. 1997). Biochemical and crystallographic analyses revealed that an LXXLL motif-containing $\alpha$-helix from coactivators interacts with a hydrophobic groove within the ligand-bound LBDs (Darimont et al. 1998; Nolte et al. 1998; Shiau et al. 1998). Importantly, the residues that comprise the hydrophobic groove are well conserved between nuclear receptors and have long been recognized as an LBD signature motif.

Initial mapping studies with the corepressor proteins revealed that the receptor interaction and repression functions are separable (Chen and Evans 1995; Horlein et al. 1995; Heinzel et al. 1997; Nagy et al. 1997), with the receptor interaction domains located toward the carboxyl terminus. Ligand binding is thought to adopt con- 
formational changes that lead to release of the corepressor and recruitment of the coactivators. However, the molecular mechanism underlying this switch remains unclear.

In this study we sought to characterize the molecular basis for the interaction between nuclear receptors and corepressors. We have identified in both SMRT and N-CoR short peptides of 19 amino acids [interaction domain (ID) 1] and 17 amino acids (ID1), with an internal signature motif (I/L)XX/I/V)I, which are sufficient for receptor interaction and ligand-induced dissociation. Sequence analyses suggest that these motifs can adopt an amphipathic $\alpha$-helical conformation, reminiscent of the signature motif LXXLL within the coactivators. Significantly, single mutations within the thyroid hormone receptor $\beta$ (TR $\beta$ ) LBD known to be involved in coactivator binding fail to bind corepressors. These results suggest an underlying mechanistic link between coactivator and corepressor binding via competition for a common or overlapping binding site.

\section{Results}

Mapping of the core receptor interaction motifs in SMRT

Previous studies have localized the receptor interaction domains to the carboxyl terminus of SMRT and N-CoR. Further studies of the SMRT receptor interaction region revealed it could be subdivided into two domains of 70 and 50 amino acids (ID1 and ID2) (Fig. 1A; see also Downes et al. 1996). Each of these domains can interact with receptors when isolated from the rest of the protein. Further examination of these two interacting domains based on homology between N-CoR and SMRT, as well as proteolysis studies of receptor corepressor complexes, suggested that the minimal interaction core may be smaller: 19 amino acids for ID2 and 17 amino acids for ID1. Two-hybrid interaction assays were established in mammalian cells in which Gal-DBD fusions of the SMRT-ID1 and SMRT-ID2 were challenged with retinoic acid receptor $\alpha(\mathrm{RAR} \alpha)$ fused to the VP16 activation domain. As shown in Figure $1 \mathrm{~B}$, both the interaction domains and the core motifs are sufficient to mediate receptor corepressor interactions and ligand-mediated release in a fashion that mimics the full-length corepressor. These peptides also function in yeast two-hybrid assays (Fig. 1C) suggesting that binding does not appear to require additional accessory factors.

\section{Mutations of the core hydrophobic residues in interaction motifs abolish receptor interaction}

Analysis of the sequences of the core motifs reveals that each one contains a putative amphipathic $\alpha$ helix (Garnier et al. 1978) (indicated by boldface underline, Fig. 2). To test the idea that the hydrophobic surface of this potential helix might form the critical surface for interaction with the receptors, we mutated these residues and tested, using the mammalian two-hybrid assay, their ability to interact with RAR. Figure 2 shows that any mutation of the core hydrophobic residues, either in clusters (M1, M3, M10) or individually (M5-M7, M12M14), abolishes interaction of the corepressor with the receptors. Interestingly, other mutations indicate that the whole domain is generally sensitive to changes or further truncations (Fig. 2, M8, M9, M15, and M16), sug-
Figure 1. Identification of minimal receptor interaction domains in SMRT. (A) A schematic representation of receptor interaction domains within the corepressor SMRT domain structure (sequence numbering corresponds to full-length human SMRT). The interaction of the different Gal-SMRT fusion constructs with RAR was evaluated using both mammalian, CV1 $(B)$ and yeast $(C)$ two-hybrid assays. Data were normalized with reference to the activity of a constitutive reporter. Transcriptional activity is expressed either as fold activation relative to Gal-DBD alone (mammalian assay) or as reporter activity (yeast). An RAR agonist at $10 \mathrm{~nm}$ (TTNPB) was used in the mammalian assay to demonstrate ligand-dependent corepressor release. Carboxyl-SMRT (C-SMRT) amino acids 2004-2517; (ID2) 2131-2201; (ID1) 2302-2352; (core ID2) 2131-2149; (core ID1) 2336-2352 (numbering as in the full-length human SMRT).

\section{A}

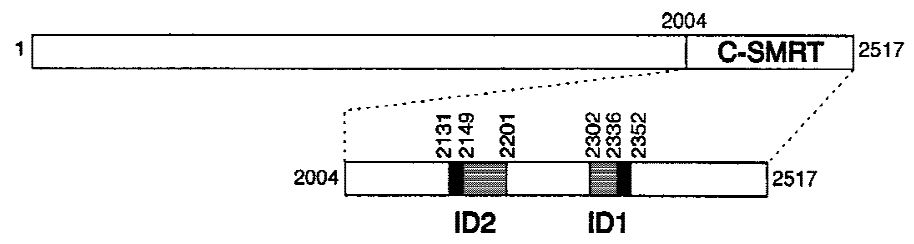

B

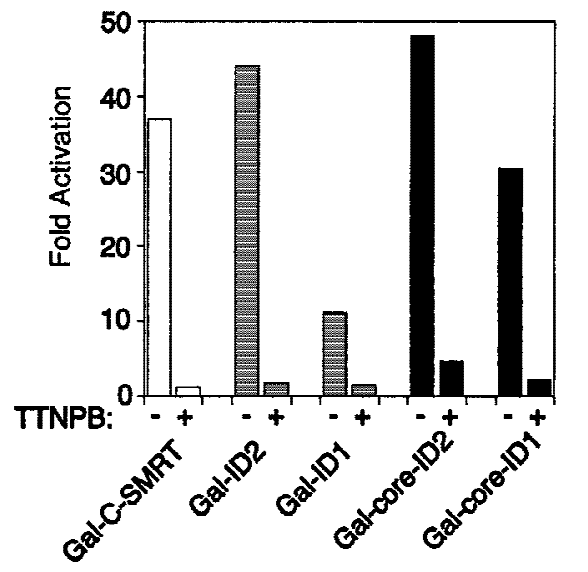

C

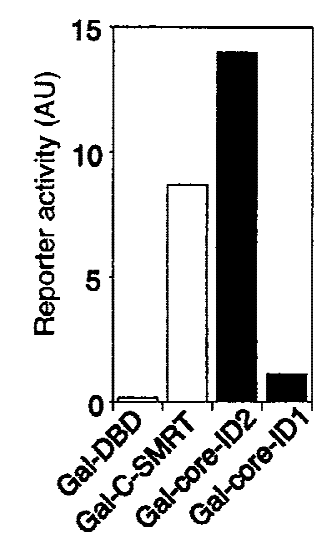


A

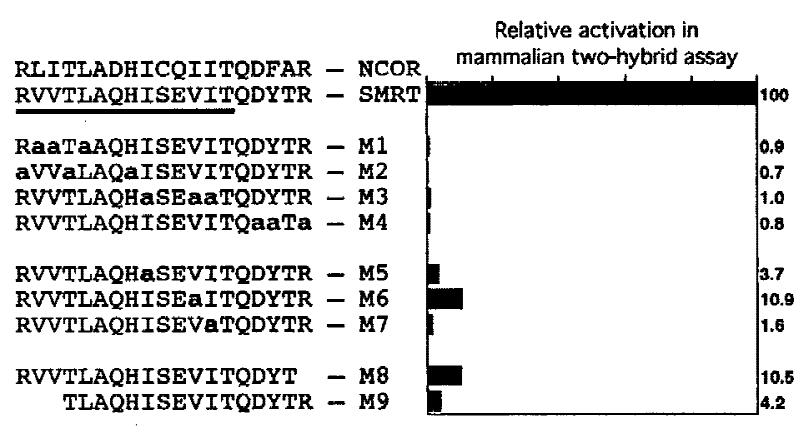

B

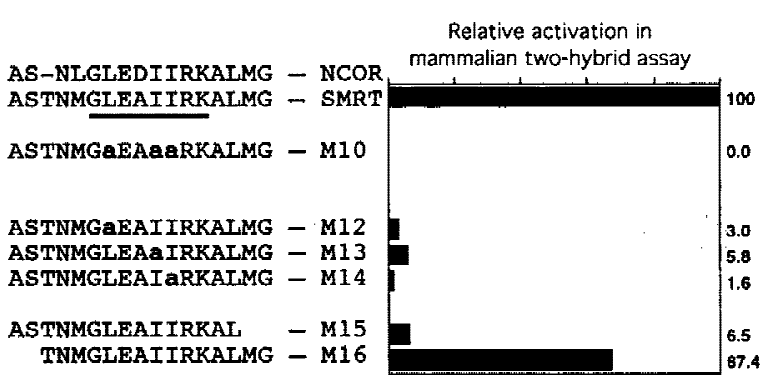

C

Relative activation in mammallan two-hybrid assay

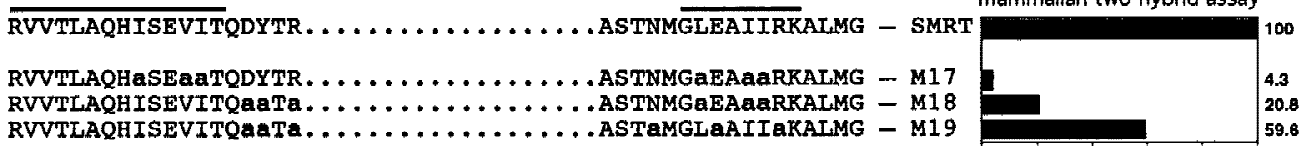

Figure 2. Mutational analyses of the minimal interaction domains ID1, ID2, and ID(1+2). (A) Mutational analyses of SMRT-ID2; $(B)$ mutational studies of SMRT-ID1; $(C)$ mutation studies of SMRT ID $(1+2)$. The bar charts show reporter activity relative to wild-type constructs. The horizontal lines indicate the extent of the helical regions predicted within ID1 and ID2. Mutants 1, 2, 3, 4, 10, 17, 18, and 19 are in the context of longer SMRT constructs [(ID2) 2131-2201; (ID1) 2302-2352; (ID[1+2]) 2131-2352]. Mutants 5-9 and 12-16 are in the context of the core IDs [(core ID2) 2131-2149; (core ID1) 2336-2352].

gesting that residues on the opposing surface of the helix can still contribute to receptor binding.

In vitro analysis of the SMRT-ID : RAR-LBD : $R X R-L B D$ complexes

To demonstrate that these SMRT-ID motifs can interact independently with receptors in vitro we assembled complexes using bacterially expressed recombinant proteins. Figure 3, A and B, shows gel filtration analyses of stoichiometric complexes of the RAR-LBD : RXR (retinoid $\underline{\mathrm{X}}$ receptor)-LBD heterodimer alone and with SMRT-ID1 and SMRT-ID2. In both cases, comparison of the gel filtration profiles with known molecular mass standards indicates a 1:1:1 stoichiometry and that the complexes are stable and nonaggregating. This was supported by light scattering techniques, which indicated that the samples were monodisperse with apparent molecular masses within $5 \mathrm{kD}$ of that expected. In the case of the RAR:RXR:SMRT-ID2 complex, equilibrium analytical ultracentrifugation analyses indicated an equilibrium between species up to a molecular mass $>65$ $\mathrm{kD}$. Interestingly, the two SMRT receptor interaction domains are significantly proteolysed unless copurified in complex with the heterodimer (data not shown). This suggests that they may be unstructured in the absence of the unliganded receptor. This is analogous to the behavior of coactivator receptor interaction domains that are also unstructured in the absence of receptor but adopt a helical conformation on binding (Nolte et al. 1998).

To characterize binding of the SMRT IDs to RAR : RXR heterodimers, an in vitro binding and peptide competition assay was established (Fig. 3E). We show that $\left[{ }^{35}\right.$ S]methionine-labeled SMRT-ID2 binds to RAR : RXR heterodimers. This binding is efficiently competed by the addition of cold wild-type peptide at micromolar concentrations (lanes 1-4). In contrast, peptides with mutations in the core hydrophobic amino acids barely compete, if at all (lanes M1-M4). This suggests that the core IDs that we have identified are both necessary and sufficient for RAR $\alpha$ binding.

To test whether there is competition between coactivator and corepressor binding to the receptor we established an assay in which we could observe coactivator binding in the absence of ligand. This was achieved by using high concentrations of ACTR (immobilized on GST resin) and RAR-LBD : RXR-LBD heterodimer. We then asked how the presence of SMRT ID1 or ID2 constructs modulates the heterodimer-coactivator interaction. Figure $3 \mathrm{C}$ shows a quantitative analysis of competitive binding between coactivator and corepressor and indicates that the addition of ligand allows restoration of the ability of the heterodimer to interact with the coactivator through the displacement of SMRT.

The identification of these motifs, which recapitulate the behavior of the entire corepressor, suggests that we have localized the critical determinants for corepressor release. If this is the case, it would suggest that we might be able to exploit these domains to create a chimeric protein that can activate transcription in the absence of ligand. Such a chimeric protein would reverse the normal signaling paradigm. We used this approach to examine the interaction between Gal-RAR and a fusion between SMRT-ID $(1+2)$ and the VP16 activation domain (Fig. 3F). Consistent with previous findings, we observed that Gal-RAR alone represses transcription (lane 2). Ad- 
A

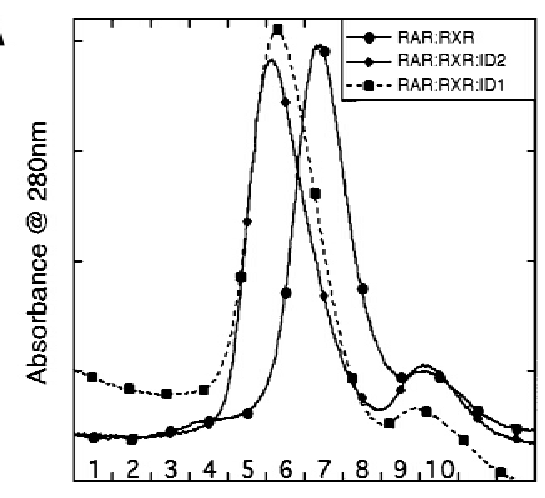

B

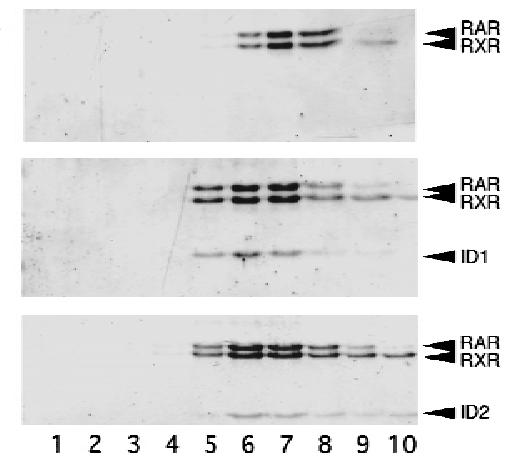

C

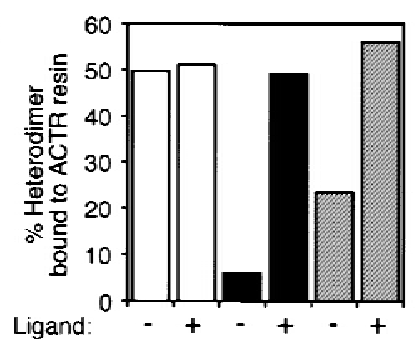

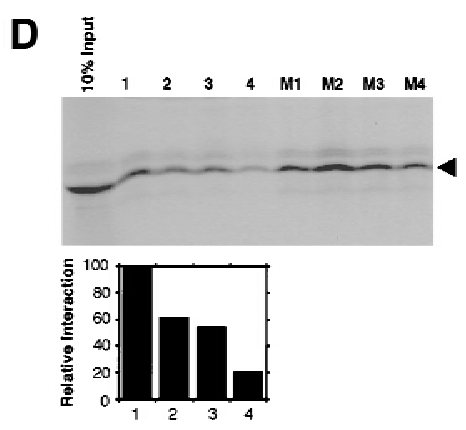

E
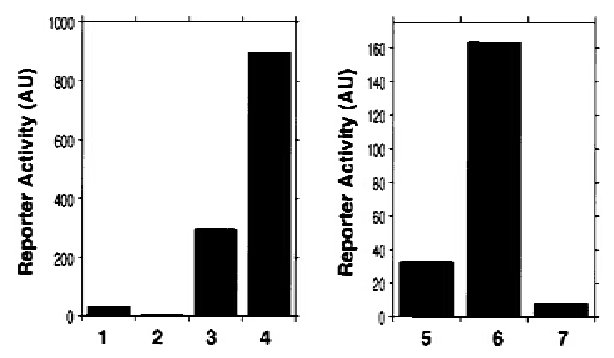

Figure 3. In vitro analysis of the SMRT-ID : RAR-LBD : RXR-LBD complexes. (A) Gel filtration profiles of purified RAR-LBD : RXR-LBD heterodimers alone and with SMRTID1 [(SID1) amino acids 2202-2352], SMRT-ID2 [(SID2) 2098-2201]. (B) SDS-PAGE corresponding to $A$. Fraction numbers are indicated below both the profiles and gels. Note the relative elution volumes for the differently sized complexes. The heterodimer is smallest and therefore retarded most by the column. In each case, the elution profile and Coomassie staining in the gel are consistent with a 1:1:1 complex. This stoichiometry was supported by equilibrium centrifugation and light-scattering techniques. $(C)$ Ligand facilitates the exchange of cofactors. The RAR agonist TTNPB increases the dissociation of SMRT-IDs from the RAR : RXR : SID1 (hatched bars) and RAR : RXR : SID2 (solid bars) complexes. This results in enhanced binding of RAR : RXR (open bars) to the GST-ACTR-ID resin. (D) In vitro competition assay with SMRT-ID2 (arrowhead) wildtype and mutant peptides. Radiolabeled ID2 was bound to GST-RXR-LBD : RAR-LBD heterodimers as described in Materials and Methods. This complex was competitively challenged by increasing the amount of wild-type synthetic peptide (lanes 1-4 using 0, 10, 30, and $100 \mu \mathrm{M}$ peptide) or mutants $1-4$ [M1-M4 at $30 \mu \mathrm{M}$ (see Fig. 2)]. Quantitation of the wild-type competition assay is shown below. (E) (Left) SMRT minimal IDs can mediate ligand-independent activation of RAR. (Lane 1) Gal-DBD alone; (lane 2) Gal-RAR alone; (lane 3) Gal-RAR + VP-SMRT (ID1 + ID2); (lane 4) Gal-RAR + VP-SMRT (ID1 + ID2) + 10 nM TTNPB (RAR-specific agonist). (Right) Ligand-induced loss of activation of an RXR helix 12 mutant. (Lane 5) Gal-DBD alone; (lane 6) Gal-RXR mut (EK, EK) + VP-SMRT (ID1 + ID2); (lane 7) Gal-RXR mut (EK, EK) + VP-SMRT (ID1 + ID2) + 100 nM LG268 (RXR-specific agonist).

dition of VP-SMRT results in a large net activation of transcription in the absence of ligand (lane 3). Addition of ligand in this assay results in activation of the reporter gene through the normal function of liganded RAR (lane 4). To prove that ligand can release the corepressor we used an RXR helix 12 mutant (RXR-E $>\mathrm{K}, \mathrm{E}>\mathrm{K}$ ). This mutant has been shown previously to be defective in transcriptional activation but not ligand binding (Schulman et al. 1997). In this case, unliganded receptor is activated by VP-SMRT-ID (1+2) (lane 6). Addition of ligand leads to reversal of activation as a consequence of SMRT-ID release (lane 7). These data emphasize that the SMRT-IDs are necessary and sufficient for interaction with receptors as well as ligand-dependent release.

Overlap of corepressor and coactivator binding sites To address whether the same motif in SMRT-IDs is also sufficient for TR $\beta$ binding, we tested the interaction of SMRT-IDs and TR $\beta$ using the mammalian two-hybrid assay. TR $\beta$ interacts strongly with ID1 (Fig. 4A), and the strength of interaction between TR $\beta$ and the core ID1 is comparable to that of the larger ID1 (50 amino acid) segment. Both full-length and core peptide show hormoneinduced dissociation (Fig. 4A). Significantly, single mutations of the core hydrophobic residues within the ID1 core abolish interaction completely with TR $\beta$ (Fig. 4B). The finding that corepressor and coactivator binding motifs are both amphipathic $\alpha$-helices suggests that they may bind to similar or overlapping sites. The coactivator motif LXXLL has been shown to bind to a hydrophobic groove on the surface of the liganded receptor (Darimont et al. 1998; Nolte et al. 1998; Shiau et al. 1998). To directly test whether corepressor interacts with the same surface on the receptor as coactivators, we examined the 
A

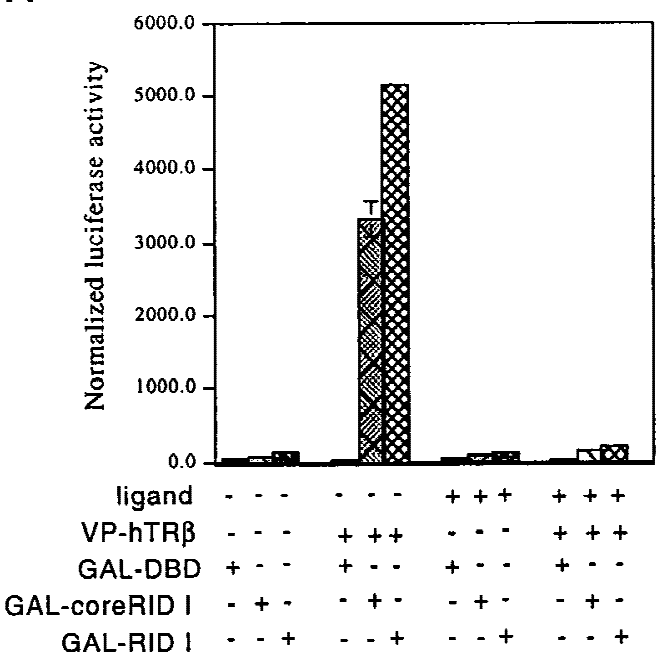

B

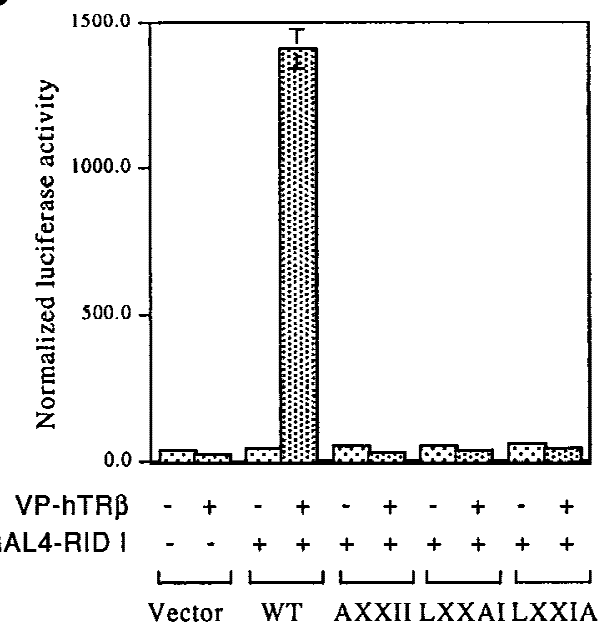

C

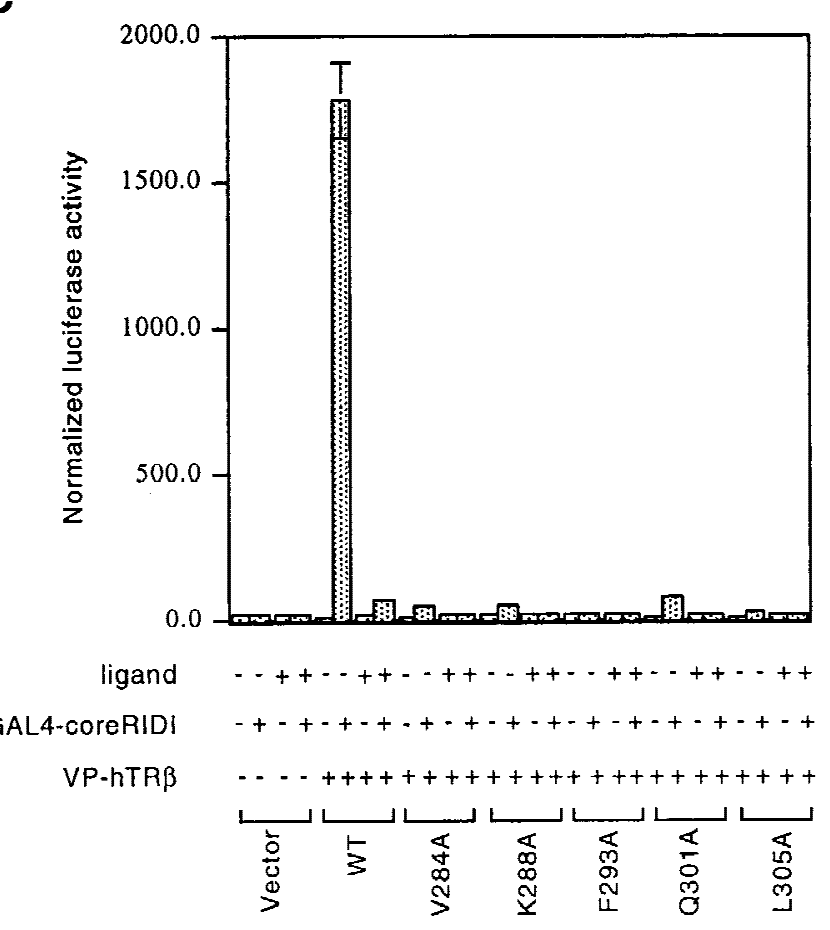

Figure 4. Residues in human (h)TR $\beta-\mathrm{LBD}$ required for coactivator interaction are also critical for corepressor binding. Mutational analysis of hTR $\beta$ and SMRT-ID1 was carried out using a mammalian twohybrid assay as described in Materials and Methods. The strength of the interactions are expressed in normalized promoter activity; the mean of three independent measurements \pm S.E. is presented. $(A) \mathrm{In}$ teraction analysis of SMRT-ID1 with hTR $\beta$. The assays were carried out in the presence or absence of $100 \mathrm{nM}$ T3. The core binding site (17 amino acids) of SMRT-ID1 is sufficient for hTR $\beta$ interaction. $(B)$ Mutations in the hydrophobic LXXII motif of SMRT ID1 abolish hTR $\beta$ interaction. Mutant residues of the core motif are indicated. (C) Mutations in hTR $\beta$ required for coactivator interaction abolish corepressor binding. Interaction analysis between the coreRID and wild-type and mutant receptor LBDs were carried out in the presence or absence of ligand (100 nM T3). ability of various TR $\beta$ mutants to interact with SMRTID1 using the mammalian two-hybrid interaction assay. We created point mutations in $\operatorname{TR} \beta$ that are known to involve in coactivator binding. Residues V284, K288, F293, Q301, and L305 are part of the groove that forms the binding surface of the coactivators (Darimont et al. 1998). Specifically, V284 of H3, F293 of H4, and L305 of $\mathrm{H} 5$ directly contact leucine residues of the signature motif LXXLL. Mutational studies with the previously described helix 1 CoR box (Horlein et al. 1995) were not considered because X-ray crystal structure clearly shows that these mutants are buried in the RAR-LBD and could not comprise a corepressor binding site (Wurtz et al. 1996). Figure 4C shows that mutations of these residues also abolished interaction with corepressors. These data indicate that these residues-284, 288, 293, 301, and 305-are critical for both coactivator and corepressor binding, leading to the surprising suggestion that their binding sites in the LBD must overlap partially.

\section{Discussion}

Perhaps the most important question concerning the function of nuclear hormone receptors is how ligand fa- 
cilitates the switch from repression to activation of gene expression. The first steps toward understanding the nature of the repressed state came with the discovery of two nuclear receptor corepressors (SMRT and N-CoR), along with the demonstration that ligand causes corepressor dissociation while simultaneously promoting the association of the coactivators (Chen and Evans 1995; Horlein et al. 1995). Significantly, both retinoid and thyroid hormone receptors remain bound to their target genes in both the repressive and active states. This means that the switch in cofactor composition can be made without dissociation of the regulatory complex.

Here we describe the identification and functional analysis of the corepressor interaction motifs that mediate recognition of unliganded receptors. Previous studies have demonstrated that coactivators interact with nuclear receptors via a short conserved association motif (Heery et al. 1997; Darimont et al. 1998; Nolte et al. 1998; Shiau et al. 1998). This work demonstrates that there is a complementary structural basis for corepression. Two short autonomous motifs in the corepressors are both necessary and sufficient for mediating corepressor binding to unliganded receptors. Both motifs are able to sense the presence of ligand by dissociating from the receptor.

The two motifs, termed ID1 and ID2 (17 and 19 amino acids, respectively), are conserved in both position and sequence between N-CoR and SMRT. Secondary structure prediction suggests that they are likely to adopt an amphipathic $\alpha$-helical conformation. This further extends the analogy with the helical coactivator LXXLL motifs. However, unlike the coactivators, it is clear that additional flanking sequences are needed for the corepressor-receptor interaction. This suggests that the binding surface for corepressors is likely to be larger than that for the coactivators.

Previous studies have suggested that mutations in the AF-2 domain of RAR result in a receptor that is able to bind ligand but is a constitutive repressor of gene expression (Chen et al. 1996b). Thus, ligand binding by itself is not sufficient to induce dissociation of the corepressor. Rather, it would appear that corepressor release requires the receptor AF-2 domain. Because coactivator association also requires the presence of the AF- 2 domains, these observations suggest mechanistic link between corepressor dissociation and coactivator binding. It has been suggested previously (Chen et al. 1996b) that there might be a competitive relationship between corepressors and coactivators, possibly by recognition of a common or overlapping binding site. We investigated this directly by mutating residues in the hydrophobic binding pocket of the receptor that has been shown to accommodate the coactivator LXXLL motif (Darimont et al. 1998; Nolte et al. 1998; Shiau et al. 1998). Remarkably, mutations at five different sites within this pocket severely disrupt corepressor binding.

Further support for there being overlapping binding sites for corepressors and coactivators is provided through the demonstration that under appropriate in vitro conditions there is a direct competition between coactivators and corepressors for the receptor's binding surface. It is clear, however, that under normal conditions the presence or absence of ligand determines whether coactivator or corepressor binding is favored. The mechanism through which ligand discriminates between the two types of cofactor remains unclear. However, three observations suggest a plausible model: (1) Coactivator binding requires a particular conformation of helix 12; (2) corepressors can bind in the absence of helix 12; and (3) helix 12 is required for ligand to induce corepressor dissociation. Taken together, these findings suggest that ligand-binding causes a conformational or dynamic change in helix 12 , resulting in the displacement of corepressor and the formation of a suitable coactivator binding surface. This in turn suggests that the corepressor binding site may overlap not only the coactivator binding site but also that of helix 12. This would fit well with the observation that all three motifs are capable of forming amphipathic $\alpha$-helical conformations (Fig. 5A). The model shown in Figure 5B is consistent with our results here, as well as the work of Zhang et al (1999), and also our previous suggestion that the AF-2 domain exists in a dynamic equilibrium between the ac-

A

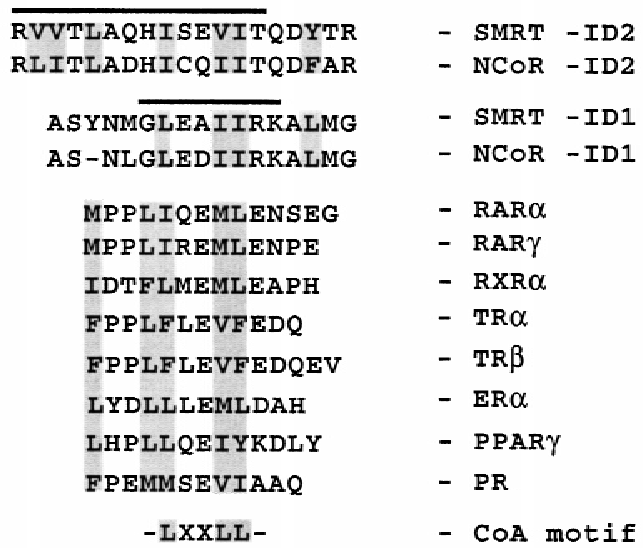

B

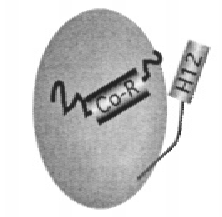

No ligand Helix 12 displaced Co-repressor bound

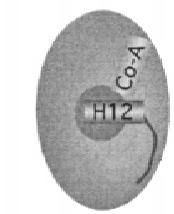

Agonist bound Helix 12 in position Co-activator bound

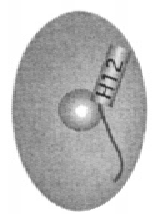

Antagonist bound Helix 12 shifted Co-activator unable to bind
Figure 5. Model for common biophysical mechanisms in coactivator and corepressor interaction with nuclear receptors. $(A)$ Conserved amphipathic helices in corepressors, helix 12 of various nuclear receptor, and the coactivator LXXLL motif. Helices are aligned with hydrophobic residues shaded gray. $(B)$ Potential role of amphipatic helices in ligand-mediated switch of nuclear receptor transcriptional activity. 
tive and the repressive conformational states (Schulman et al. 1997).

In conclusion, the identification of a corepressor signature motif provides insight into the mechanism of repressor recruitment, as well as suggesting a mechanism whereby ligand-binding switches nuclear receptors from transcriptional repressors to activators, thus explaining the key feature of hormone signaling.

\section{Materials and methods}

\section{Materials}

Chemicals were purchased from Sigma (St Louis, MO) unless indicated otherwise. The ligand LG100268 was the kind gift of Richard A. Heyman (Ligand Pharmaceuticals, San Diego, CA). TTNPB was purchased from Biomol, Inc. Mutations were generated by using the QuickChange kit (Stratagene, La Jolla,CA) according to the manufacturer's directions. Mutations were verified by sequencing.

Transient transfection experiments in mammalian cells and yeast transformations

Mammalian expression vectors used in this work have been described previously (Chen and Evans 1995). Plasmids were constructed by standard cloning techniques. Detailed information is available on request. CV1 cells were transiently transfected as described previously (Chen and Evans 1995). Luciferase activity of each sample was normalized with reference to the level of $\beta$-galactosidase activity of a control reporter construct. Each transfection was carried out in triplicate at least three times. Percentage of maximal induction of the reporter gene or normalized luciferase activity is presented as indicated in Figures $1 \mathrm{~B}, 2,3 \mathrm{~F}$, and 4 . Yeast transfromation and $\beta$-galactosidase assays were carried out according to the manufacturer's instructions (Clontech).

\section{Gel filtration and competition assays}

For the gel filtration assays histidine-tagged RAR-LBD and RXR-LBD proteins were coexpressed in bacteria and purified as described previously (Li et al. 1997). SMRT constructs were also expressed using the T7 expression system. Protein complexes were made by mixing resuspended cell pellets prior to lysis by sonication in a buffer containing $50 \mathrm{mM}$ HEPES ( $\mathrm{pH} 8.0$ ), 150 $\mathrm{mm} \mathrm{NaCl}, 2.5 \mathrm{mM} \mathrm{CaCl}_{2}, 20 \mathrm{~mm}$ imidazole, and $1 \mathrm{~mm}$ AEBSF (Boehringer Mannheim/Melford). The histidine-tagged proteins were bound to Ni-NTA agarose resin (Qiagen) and, after repeated washing, eluted using lysis buffer containing $250 \mathrm{~mm}$ imidazole. Fractions containing the receptor or receptor-cofactor complexes were pooled, concentrated (Amicon-stirred cell), and further purified by anion exchange chromatography [200 $\mathrm{mm} \mathrm{NaCl}$ elution from Poros PI resin (PE Biosystems)]. Complexes were purified from excess components using a Superdex S200 gel filtration column (Pharmacia).

For the competition assays, GST-RXR and His-RAR were coexpressed as described previously (Li et al. 1997). The cells were lysed by sonication and the heterodimer purified using glutathione-Sepharose (Pharmacia) in buffer containing $50 \mathrm{~mm}$ Tris $(\mathrm{pH} 8), 1 \%$ Triton $\mathrm{X}-100$, and $1 \mathrm{~mm}$ AEBSF. $\left[{ }^{35}\right.$ S]methionine-labeled SMRT-ID proteins were prepared by in vitro transcription/translation (Promega). Peptides were either synthesized in-house or purchased from Peptides Products or Genosys. Competition assays were performed in buffer containing $50 \mathrm{~mm}$
Tris ( $\mathrm{pH} 7.4$ ), $0.5 \%$ Triton $\mathrm{X}-100$, and $1 \mathrm{mg} / \mathrm{ml}$ BSA. GST pulldowns were performed using GST-RXR-LBD : RAR-LBD immobilized on glutathione-Sepharose resin (Pharmacia). Gels were quantitated using an image plate scanner (Molecular Dynamics).

\section{Acknowledgments}

We are most grateful to Drs. Yaki Barak, Bruce Blumberg, Louise Fairall, Barry Forman, Michael Moore, and Enrique Saez for helpful discussions; to Cynthia Simon, Jackie Alvarez, and Henry Juguilon for invaluable technical help; to Dr. Richard Heyman for reagents; and to Elaine Stevens for administrative assistance. L.N. is supported by a Special Fellowship from the Leukemia Society of America. H.-Y. Kao is a Fellow of the Leukemia Society of America. R.M.E is an Investigator of the Howard Hughes Medical Institute and March of Dimes Chair of Molecular and Developmental Biology at the Salk Institute. This work was supported by HHMI and National Institutes of Health grants GM26444 and HD27183.

The publication costs of this article were defrayed in part by payment of page charges. This article must therefore be hereby marked "advertisement" in accordance with 18 USC section 1734 solely to indicate this fact.

\section{References}

Alland, L., R. Muhle, H. Hou, Jr., J. Potes, L. Chin, N. SchreiberAgus, and R.A. DePinho. 1997. Role for N-CoR and histone deacetylase in Sin3-mediated transcriptional repression. $\mathrm{Na}$ ture 387: 49-55.

Blanco, J.C., S. Minucci, J. Lu, X.J. Yang, K.K. Walker, H. Chen, R.M. Evans, Y. Nakatani, and K. Ozato. 1998. The histone acetylase PCAF is a nuclear receptor coactivator. Genes \& Dev. 12: 1638-1651.

Chen, H., R. Lin, R. Schiltz, D. Chakravarti, A. Nash, L. Nagy, M. Privalsky, Y. Nakatani, and R. Evans. 1997. Nuclear receptor coactivator ACTR is a novel histone acetyltransferase and forms a multimeric activation complex with $\mathrm{P} / \mathrm{CAF}$ and CBP/p300. Cell 90: 569-580.

Chen, J.D. and R.M. Evans. 1995. A transcriptional co-repressor that interacts with nuclear hormone receptors. Nature 377: 454-457.

Chen, J., J. Clifford, C. Zusi, J. Starrett, D. Tortolani, J. Ostrowski, P. Reczek, P. Chambon, and H. Gronemeyer. 1996a. Two distinct actions of retinoid-receptor ligands. Nature 382: 819-822.

Chen, J.D., K. Umesono, and R.M. Evans. 1996b. SMRT isoforms mediate repression and anti-repression of nuclear receptor heterodimers. Proc. Natl. Acad. Sci. 93: 7567-7571.

Darimont, B., R. Wagner, J. Apriletti, M. Stallcup, P. Kushner, J. Baxter, R. Fletterick, and K. Yamamoto. 1998. Structure and specificity of nuclear receptor-coactivator interactions. Genes \& Dev. 12: 3343-3356.

Downes, M., L.J. Burke, P.J. Bailey, and G.E. Muscat. 1996. Two receptor interaction domains in the corepressor, N-CoR/ RIP13, are required for an efficient interaction with Rev-erbA alpha and RVR: Physical association is dependent on the E region of the orphan receptors. Nucleic Acids Res. 24: 4379-4386.

Garnier, J., D.J. Osguthorpe, and B. Robson. 1978. Analysis of the accuracy and implications of simple methods for predicting the secondary structure of globular proteins. J. Mol. Biol.120: 97-120. 
Nagy et al.

Hassig, C.A., T.C. Fleischer, A.N. Billin, S.L. Schreiber, and D.E. Ayer. 1997. Histone deacetylase activity is required for full transcriptional repression by $\mathrm{mSin} 3 \mathrm{~A}$. Cell 89: 341-347.

Heery, D., E. Kalkhoven, S. Hoare, and M. Parker. 1997. A signature motif in transcriptional co-activators mediates binding to nuclear receptors. Nature 387: 733-736.

Heinzel, T., R.M. Lavinsky, T.M. Mullen, M. Soderstrom, C.D. Laherty, J. Torchia, W.M. Yang, G. Brard, S.D. Ngo, J.R. Davie et al. 1997. A complex containing N-CoR, mSin3 and histone deacetylase mediates transcriptional repression. $\mathrm{Na}$ ture 387: 43-48.

Hong, H., K. Kohli, A. Trivedi, D.L. Johnson, and M.R. Stallcup. 1996. GRIP1, a novel mouse protein that serves as a transcriptional coactivator in yeast for the hormone binding domains of steroid receptors. Proc. Natl. Acad. Sci. 93: 49484952.

Horlein, A.J., A.M. Naar, T. Heinzel, J. Torchia, B. Gloss, R. Kurokawa, A. Ryan, Y. Kamei, M. Soderstrom, C.K. Glass et al. 1995. Ligand-independent repression by the thyroid hormone receptor mediated by a nuclear receptor corepressor. Nature 377: 397-404.

Kamei, K., L. Xu, T. Heinzel, J. Torchia, R. Kurokawa, B. Gloss, S.-C. Lin, R.A. Heyman, D.W. Rose, C.K. Glass, and M.G. Rosenfeld. 1996. A CBP Integrator complex mediates transcriptional activation and AP-1 inhibition by nuclear receptors. Cell 85: 403-414.

Laherty, C.D., W.M. Yang, J.M. Sun, J.R. Davie, E. Seto, and R.N. Eisenman. 1997. Histone deacetylase associated with the $\mathrm{mSin} 3$ corepressor mediate Mad transcriptional repression. Cell 89: 349-356.

Li, C., J. Schwabe, E. Banayo, and R. Evans. 1997. Coexpression of nuclear receptor partners increases their solubility and biological activities. Proc. Natl. Acad. Sci. 94: 2278-2283.

Mangelsdorf, D.J. and R.M. Evans. 1995. The RXR heterodimers and orphan receptors. Cell 83: 841-850.

Mangelsdorf, D.J., C. Thummel, M. Beato, P. Herrlich, G. Schutz, K. Umesono, B. Blumberg, P. Kastner, M. Mark, P. Chambon, and R.M. Evans. 1995. The nuclear receptor superfamily: The second decade. Cell 83: 835-839.

Nagy, L., H.Y. Kao, D. Chakravarti, R.J. Lin, C.A. Hassig, D.E. Ayer, S.L. Schreiber, and R.M. Evans. 1997. Nuclear receptor repression mediated by a complex containing SMRT, mSin3A, and histone deacetylase. Cell 89: 373-380.

Nolte, R., G. Wisely, S. Westin, J. Cobb, M. Lambert, R. Kurokawa, M. Rosenfeld, T. Willson, C. Glass, and M. Milburn. 1998. Ligand binding and co-activator assembly of the peroxisome proliferator-activated receptor. Nature 395: 137143.

Onate, S., S. Tsai, M. Tsai, and B. O'Malley. 1995. Sequence and characterization of a co-activator for the steroid hormone receptor superfamily. Science 270: 1354-1357.

Schulman, I., C. Li, J.W.R. Schwabe, and R. Evans. 1997. The phantom ligand effect: Allosteric control of transcription by the retinoid X receptor. Genes \& Dev. 11: 299-308.

Shiau, A., D. Barstad, P. Loria, L. Cheng, P. Kushner, D. Agard, and G. Greene. 1998. The structural basis of estrogen receptor/coactivator recognition and the antagonism of this interaction by tamoxifen. Cell 95: 927-937.

Torchia, J., D. Rose, J. Inostroza, Y. Kamei, S. Westin, C. Glass, and M. Rosenfeld. 1997. The transcriptional co-activator $\mathrm{p} / \mathrm{CIP}$ binds CBP and mediates nuclear receptor function. Nature 387: 677-684.

Wurtz, J.-M., W. Bourguet, J.-P. Renaud, V. Vivat, P. Chambon, D. Moras, and H. Gronemeyer. 1996. A canonical structure for the ligand-binding domain of nuclear receptors. Nat. Struct. Biol. 3: 87-94.
Yao, T.-P., G. Ku, N. Zhou, R. Scully, and D.M. Livingston. 1996. The nuclear hormone receptor coactivator SRC-1 is a specific target of p300. Proc. Nat1. Acad. Sci. 93: 1062610631.

Zhang, Y., R. Iratni, H. Erdjument-Bromage, P. Tempst, and D. Reinberg. 1997. Histone deacetylases and SAP18, a novel polypeptide, are components of a human Sin 3 complex. Cell 89: $357-364$.

Zhang, J., X. Hu, and M.A. Lazar. 1999. A novel role for helix 12 of retinoid X receptor in regulating repression. Mol. Cell. Biol. 19: 6448-6457. 


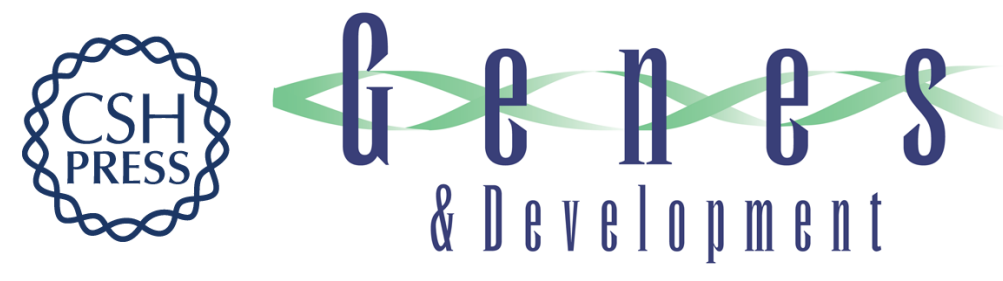

\section{Mechanism of corepressor binding and release from nuclear hormone receptors}

Laszlo Nagy, Hung-Ying Kao, James D. Love, et al.

Genes Dev. 1999, 13:

References This article cites 29 articles, 9 of which can be accessed free at:

http://genesdev.cshlp.org/content/13/24/3209.full.html\#ref-list-1

License

Email Alerting
Service $\begin{aligned} & \text { Receive free email alerts when new articles cite this article - sign up in the box at the top } \\ & \text { right corner of the article or click here. }\end{aligned}$

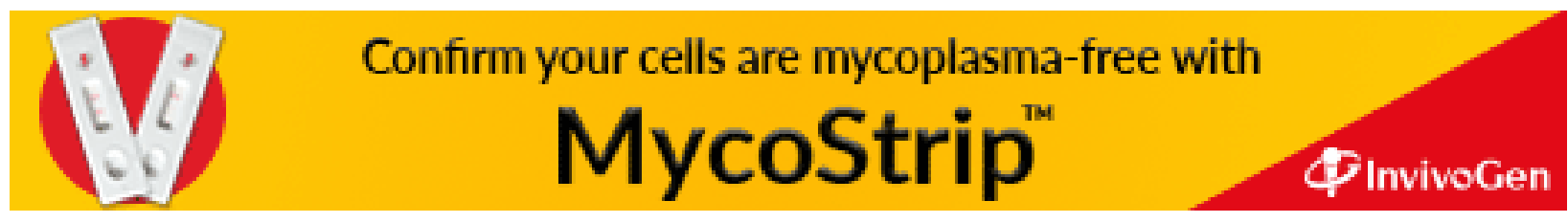

